\title{
FPGA Implementation of MIMO System using Xilinx System for Video Transmission
}

\author{
Shreya Kaushal ${ }^{1}$, Gurjinder Kaur ${ }^{2}$ \\ ${ }^{1}$ (Ece, Gurukul Vidyapeeth, India) \\ ${ }^{2}$ (Ece, Gurukul Vidyapeeth, India)
}

\begin{abstract}
In this paper, we have introduced an architecture for real-time video transmission over multipleinput multiple-output (MIMO) wireless communication systems. We employ the $2 \times 1$ and $2 \times 2$ Alamouti MIMO technique to develop a transmission system and implement the design on FPGA using Xilinx System using the modeling and simulation capabilities of MATLAB. Furthermore, according to the contribution to the reconstructed video quality, we apply unequal error protection strategy using STBC space-time codes for each video bitstream. We present a solution using FPGA as target platform (instead of software), for implementing algorithms for transmission of educational videos using MIMO systems. In our proposed system, we have provided the system performance of a joint MPEG-2 coding scheme with convolutional channel coding and space time block coding (STBC) techniques, associated with suitable modulation method (QAM and BPSK), for video data transmission over a wireless MIMO system with Rayleigh fading noises. Simulation results show the quality of the reconstructed image can be significantly improved over only using space time coding. The comparison has been done on the basis of quality of reproduced image by measuring image PSNR based on SNR and BER value for different system model is realized over Xilinx Virtex-4 XC4VFX100FFG1517-11C FPGA based WRAP board.
\end{abstract}

Keywords: FPGA (Field Programmable Gate Arrays); STBC (Space Time Block Coding), Bit error rate(BER), FPGA, Peak to signal noise ratio (PSNR), Multiple input multiple output (MIMO), WRAP board, Joint source channel coding, Space time block coding(STBC).

\section{Introduction}

To meet rapidly increasing demands for the multimedia services and better quality of service over wireless communication systems, MIMO (Multiple Output Multiple Input) systems have been proposed which promise enhanced system capacity, throughput and data reliability[1]. MIMO has been defined as two or more unique radio signals, in the same radio channel, where each signal carries different digital information and/or two or more radio signals which use beam-forming, receive combining, and spatial multiplexing. Spectral efficiency, robustness and implementation complexity are the most important issues that should be taken into account for the design of new physical layer technologies. As conventional methods like using more bandwidth or higher order modulation types are limited, new methods of using the transmission channel have to be used.

Depending on the research requirements, MIMO testbeds can generally be characterized into three types, software-defined, high performance real time based, and FPGA or digital signal processor based[2]. However, when the intended testbeds use includes developing hardware algorithm implementations, then inclusion of high performance FPGA or DSP cores is required to facilitate real-time processing. FPGA design and implementation of MIMO test bed has received a significant attention in recent years. Wireless testbeds have traditionally been implemented on general-purpose, sequential, Digital Signal Processors (DSP) or on Application Specific Integrated Circuits (ASIC). Enhanced algorithms, which are generally highly parallelizable, and higher data transmission rates can burden DSP beyond its capacity for real-time processing. MIMO systems can be used to increase system capacity as well as data reliability in wireless communication systems [2]. Research has been conducted in developing space-time codes [15] for transmission over MIMO systems.

\section{Warplab Framework}

In our research work, we present WARPLab, a framework which allows rapid prototyping of algorithms for wireless communications by combining the ease of MATLAB with the capabilities of the Wireless Open-Access Research Platform (WARP). The WARPLab framework provides the software necessary for easy interaction with the WARP hardware directly from the MATLAB workspace. The user M-Code is the code the user writes in order to generate the digital baseband samples to be transmitted. Using the functions in the WARPLab reference M-Code the user can control, directly from MATLAB, the transmission and reception of these samples in real-time RF over-the-air using WARP nodes[3]. 


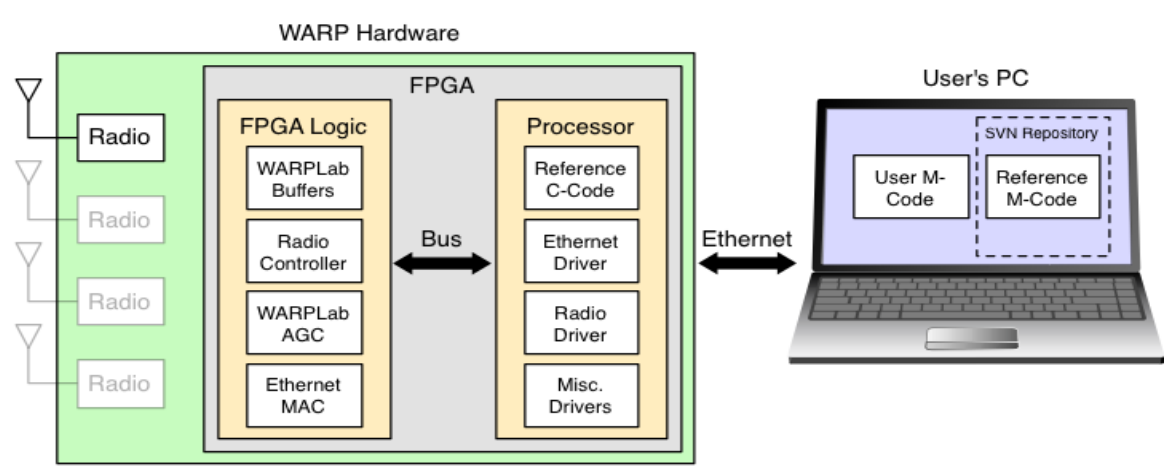

Figure1. WARP NODE Design Architecture

WARP is being actively used for research in many areas like power management, architectures for wireless receivers, physical layer algorithms, access protocols, routing and cognitive radios. The Wireless OpenAccess Research Platform (WARP) is a scalable and extensible programmable wireless platform, built from the ground up to prototype advanced wireless networks[4]. WARP combines high-performance programmable hardware with an open-source repository of reference designs and support materials. WARPLab is a framework for rapid physical layer prototyping that allows for coordination of arbitrary combinations of single and multiantenna transmit and receive nodes. The extensible framework gives users the flexibility to develop and deploy large arrays of nodes to meet any application or research need.

\section{System Design And Principal}

We are interested in joint source-channel coding with modulation scheme design under the channel capacity constraint consideration in a MIMO system. We have applied the transmission system design method for digital transmission of image over noisy channel. To transmit a given video bit stream efficiently, we propose a joint source-channel coding system. In this system, the video is undergone through convolution channel coding to protect the bit stream by the error correction encoder. In this proposed system, the video sequence is first source coded by a MPEG2 scheme (Sikora, 1997). In order to reduce the system complexity of decoding, after the source coding stage, we use convolutional code and STBC in channel coding. The interleaver is adopted which is effected resisting burst error in wireless channel. There are two modulation techniques employed to be selected, BPSK or QAM. The channel capacity is limited to one bit/transmission. In the proposed system, there are three MPEG2 video coding rates adopted and the resulted compressed video frames compared with the original test video. The source coding scheme is MPEG2 format and there are $145 \times 125$ pixels in every frame.

\section{Evaluation Parameters}

In image and video processing, the most commonly used measurements for estimating the difference between two images are mean squared error (MSE) and peak signal-to-noise ratio(PSNR)[5]. PSNR, is a term for ratio between the maximum possible power of a signal and the power of corrupting noise that affects the fidelity of its representation. Because many signals have a wide dynamic range PSNR is usually expressed in terms of logarithmic decibel scale[8]. The PSNR is most commonly used as a measure of quality of reconstruction of lossy image. The signal in his case is the original data. For color images with three RGB values per pixel, the definition of PSNR is the same except the MSE is the sum over all squared value differences divided by image size and by three.

When the two images are identical, the MSE will be zero. For this value the PSNR is undefined[9].The comparison among these two system configuration is also done by calculating the BER. It is most easily defined via the mean squared error (MSE) which for two monochrome images and G where one of the images is considered a noisy approximation of the other is defined as[6]:

$$
\mathrm{MSE}=\frac{1}{\mathrm{MN}} \sum_{\mathrm{i}=1}^{\mathrm{M}} \sum_{\mathrm{j}=1}^{\mathrm{N}}[\mathrm{F}(\mathrm{i}, \mathrm{j})-\mathrm{G}(\mathrm{i}, \mathrm{j})]^{\wedge} 2
$$

The PSNR is defined as:

$\operatorname{PSNR}(\mathrm{dB})=10 * \log 10\left(\left[(\text { maximumvalue })^{\wedge} 2\right] / \mathrm{MSE}\right)$

Or

$\operatorname{PSNR}(\mathrm{dB})=20 * \log 10([$ MaximumValue/Sqrt(MSE) $])$ 
Table1. Performance Evaluation of PSNR and SNR for 2x1 MIMO System

\begin{tabular}{|l|l|l|l|l|l|l|l|l|}
\hline SNR & 1 & 2 & 3 & 4 & 5 & 6 & 7 & 8 \\
\hline PSNR & 12.9 & 13.5 & 14.5 & 15.5 & 16.3 & 17.7 & 18.4 & 19.9 \\
\hline
\end{tabular}

Table2. Performance Evaluation of PSNR and SNR for 2x2 MIMO System

\begin{tabular}{|c|c|c|c|c|c|c|c|c|c|}
\hline SNR & 1 & 2 & 3 & 4 & 5 & 6 & 7 & 8 & 9 \\
\hline PSNR & 14.99 & 15.064 & 17.04 & 18.85 & 19.47 & 21.456 & 22.876 & 24.27 & 25.86 \\
\hline
\end{tabular}

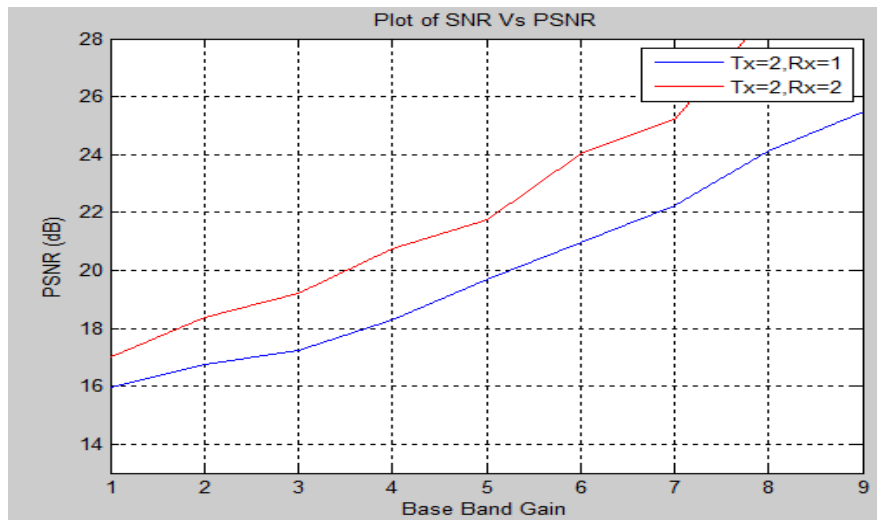

Fig.2 SNR Vs PSNR Plot

\section{Channel Coding -Convolutional Coding And Space-Time Block Coding}

In order to reduce the channel error effect and to improve the system performance while transmitting video signals over wireless channel, we have employed the convolutional encoder and maximum-likelihood Viterbi hard decision decoder for channel error correction. Figure 5 shows a typical $1 / 2$ recursive systematic convolutional (RSC) code scheme with generator function[7]. After the convolutional encoding, the processed data is fed into a random interleaver to reduce burst error effect in wireless channel. The convolutional coding rates provided in this proposed system are set as $2 / 5,1 / 2$, and $2 / 3$, respectively. The channel coding rate selected is corresponding to the MPEG2 source coding rate to satisfy the channel capacity limitation to one bit/transmission. For the system simulation, we have adopted modulation types: QAM and BPSK. In order to receive a decent quality video sequence over wireless MIMO system with good coding gain real time, we have selected convolutional code and space time block code (STBC) for the channel coding[8]. It has been known that a transmission system with antenna diversity can achieve reliable communication over wireless channel. Antenna diversity is achieved by employing spatially separated antennas at the transmitter and/or receiver. The advantage with multiple antennas scheme is that it results in a drastic increase in the channel capacity.

Table3.Parameters showing convolutional coding rates

\begin{tabular}{|c|c|c|c|}
\hline Type & Transmission rate & Channel coding & Source coding rate \\
\hline A & 0.8743 bit & $2 / 5$ & $0.3256 \mathrm{bpp}$ \\
\hline B & $0.9985 \mathrm{bit}$ & $2 / 3$ & $0.7389 \mathrm{bpp}$ \\
\hline
\end{tabular}

To realize the channel coding rate effect under wireless Rayleigh fading channel with AWGN noise conditions, we have performed the experiment for $2 \times 2$ system antenna structure with three convolutional coding rates: $2 / 5$ and 2/3, respectively. The resulted system performance is shown in Figure 7.

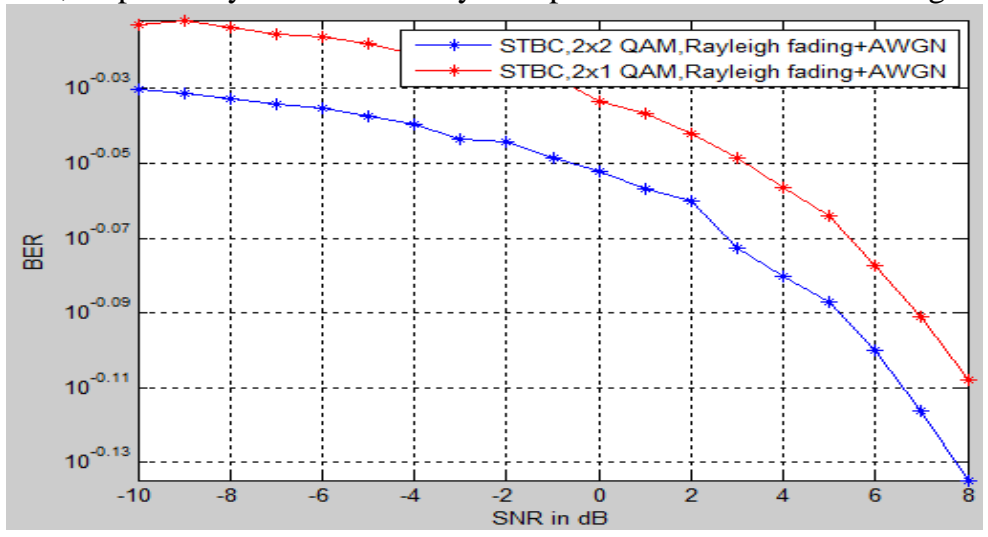

Fig.3 The BER performance comparision of STBC configuration in Rayleigh fading channel (2/5 convolution code rate using BPSK Modulation) 


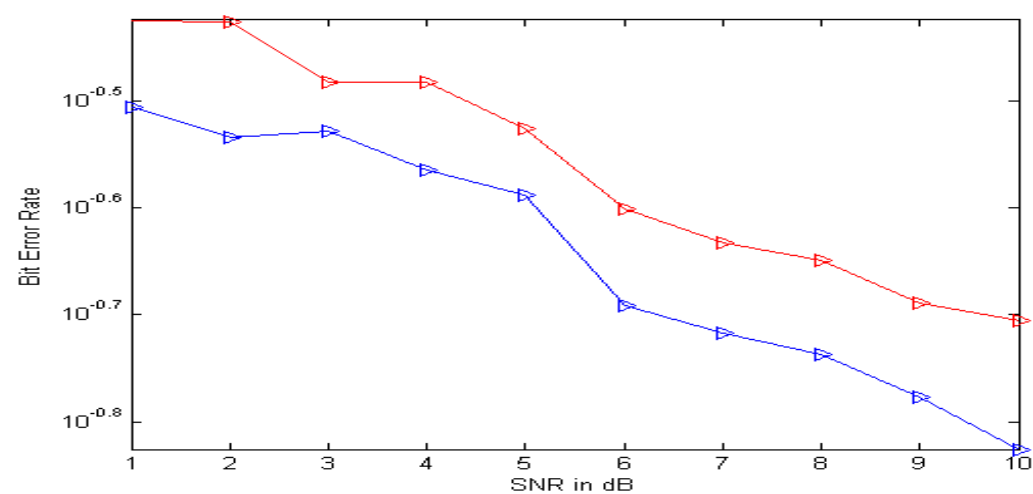

Fig.4 The BER performance comparison of STBC configuration in Rayleigh fading channel (2/3 convolution code rate using QAM Modulation)

\section{Results}

This paper shows the bit error ratio (BER) performance as a function of the signal to noise ratio (SNR) per bit of the different versions of the system. The PHY protocol maps the received signal strength and decides the appropriate transmission rate. The simulation results of the PSNR and various SNR for proposed method on the different system models such as $(2 \mathrm{Tx}, 1 \mathrm{Rx})$ and $(2 \mathrm{Tx}, 2 \mathrm{Rx})$ antenna systems using QAM are plotted. To realize the channel coding rate effect under wireless Rayleigh fading channel with AWGN noise conditions, we have performed the experiment for $2 \times 2$ system antenna structure with three convolutional coding rates: $2 / 5$ and $2 / 3$, respectively, rate $2 / 3$ convolutional coded system is with the worst bit error rate (BER) performance, where rate $2 / 5$ convolutional coded system shown the best BER performance at the same SNR conditions. A higher number of $\mathrm{B}$ frames provides better quality for the same bit rate.

\section{Conclusion}

The transmission of a MATLAB video clip is evaluated in the described testbed. The source station continuously transmits frames of a video clip over the air. The destination station receives the video and decodes it using MATLAB. The demo consists of three sequential phases. Xilinx is a system level modeling tool that facilitates FPGA hardware design by extending Matlab in numerous ways in order to provide a powerful modeling environment. Rates assigned to MPEG-2 source code and convolutional channel code as well as space-time block code schemes are based on the feedback information from Performance Control Unit (PCU) under system channel capacity limitation, which ensures the proposed system achieved the best performance compared to a conventional designed system. Experimental results have demonstrated that the proposed scheme can be an excellent alternative to achieve desired tradeoff between the reconstructed video quality and the transmission efficiency.

\section{References}

[1]. S.Gupta, S.Mittal, S.Dasgupta, A.Mittal. "MIMO Systems For Ensuring Multimedia QoS Over Scarce Resource Wireless Networks." in ACM International Conference On Advance Computing, India 2008.

[2]. D. Gesbert, M. Shafi, D. Shiu, P.J. Smith, A. Naguib. "From theory to practice: an overview of MIMO space-time coded wireless systems." IEEE Journal on Selected Areas in Communications, Vol. 21,no. 3, pp. 281-302, 2003

[3]. WARPLab framework. 2010. URL http://warp.rice.edu/WARPLab.

[4]. "WARPRepository:http://warp.rice.edu/trac"

[5]. http://en.wikipedia.org/wiki/user:renatol/PSNR"

[6]. http://multimediatechnology.google.com/svnhistory/r7/trunk/lab2jpeg/info/PSNR.pdf

[7]. Z.Wang and A.C.Bovik, "A universal image Quality index", IEEE Signal Processing Letters, vol 9, no 3,2002.

[8]. D.Gesbert, M.Shafi, "From Theory To Practice: an overview of MIMO Space time coded wireless systems", IEEE Journal On Selected Areas In Communication, vol 21, no 3, pp 281-302,2003

[9]. Dales Bates, Soren Henriksen, "A 4x4 FPGA based wireless testebed for LTE applications", IEEE $19^{\text {th }}$ International Symposium, pp 1-5, 2008.

[10]. Duan Jinghong, Deng Yaling Kun, "Develeopment Of image processing system based on DSP and FPGA", International Conference On Electronic Measurements And Instruments, pp 791-794, 2007 